\title{
Anderson Localization in Quantum Chaos: Scaling and Universality
}

\author{
A.M. GarcíA-García \\ Physics Department, Princeton University, Princeton, New Jersey 08544, USA \\ and The Abdus Salam International Centre for Theoretical Physics \\ P.O.B. 586, 34100 Trieste, Italy

\section{AND J. WANG} \\ Temasek Laboratories, National University of Singapore, 117542 Singapore \\ and
}

Beijing-Hong Kong-Singapore Joint Center for Nonlinear and Complex Systems National University of Singapore, 117542 Singapore

\begin{abstract}
The one-parameter scaling theory is a powerful tool to investigate Anderson localization effects in disordered systems. In this paper we show that this theory can be adapted to the context of quantum chaos provided that the classical phase space is homogeneous, not mixed. The localization problem in this case is defined in momentum, not in real space. We then employ the one-parameter scaling theory to: (a) propose a precise characterization of the type of classical dynamics related to the Wigner-Dyson and Poisson statistics which also predicts in which situations Anderson localization corrections invalidate the relation between classical chaos and random matrix theory encoded in the Bohigas-Giannoni-Schmit conjecture, (b) to identify the universality class associated with the metal-insulator transition in quantum chaos. In low dimensions it is characterized by classical superdiffusion, in higher dimensions it has in general a quantum origin as in the case of disordered systems. We illustrate these two cases by studying $1 d$ kicked rotors with non-analytical potentials and a $3 d$ kicked rotor with a smooth potential.
\end{abstract}

PACS numbers: 72.15.Rn, 71.30.+h, 05.45.Df, 05.40.-a

\section{Introduction}

The Bohigas-Giannoni-Schmit (BGS) conjecture [1] states that quantum spectra of classically chaotic systems are universally correlated according to the Wigner-Dyson (WD) random matrix ensembles. Therefore, for scales of time com- 
parable to the Heisenberg time the quantum system evolves to a state where all dynamical features are washed out, and the whole phase space available is occupied. Eigenstates are fully delocalized and the spectral correlations are dictated by symmetry only. For the sake of completeness we mention that the relation between level repulsion and classical chaos was noted previously in [2, 3]. However, we stick to the term "BGS conjecture" through the paper since Bohigas and co-workers were the first to claim that (a) this relation was universal, (b) spectral correlations are described by random matrix theory. From our point of view this is a qualitative difference from the findings of Refs. $[2,3]$.

Similarly, according to the Berry-Tabor-Gutzwiller (BTG) conjecture [4], the spectral correlations of classically integrable systems are well described by Poisson statistics. According to the Noether theorem there is a conserved quantity (generalized momentum) for each classical symmetry. Integrability assures that the number of these conserved momenta is equal to the dimensionality of the space. In quantum mechanics each conserved quantity leads to a good quantum number. A given quantum state is thus characterized by a unique set of good momentum quantum numbers. Since different states are characterized by a different set of quantum numbers, the spectrum is not correlated. In other words, in integrable systems the Hamiltonian is diagonal in a basis of momentum eigenstates.

The influence of these two conjectures in the establishment and development of quantum chaos is hard to overestimate. The reason is simple: Comparatively it is straightforward to determine whether the classical dynamics is chaotic or integrable. With this input one can predict the quantum transport properties of the system even without a full consideration of the specific details of the Hamiltonian.

However, exceptions are well documented in the literature, among others we cite arithmetic billiards [5], Harper model [6], and the ubiquitous kicked rotor [7], namely, a free particle in a circle which experiences periodic kicks of amplitude given by a periodic smooth potential,

$$
\mathcal{H}=\frac{p^{2}}{2}-K \cos (q) \sum_{n} \delta(t-T n) .
$$

For short time scales, both quantum and classical motions are diffusive in momentum space. However, quantum diffusion is eventually arrested due to destructive interference effects that localize eigenstates in momentum space. In this limit spectral correlations are described by Poisson but not by WD statistics. This counter-intuitive feature, usually referred to as dynamical localization [7], was fully understood [8] after mapping the kicked rotor problem onto a short range one-dimensional disordered system where localization is well established.

Deviations from the BGS conjecture are also expected [9] for eigenvalue separations $\delta E \sim \hbar / t_{\mathrm{E}}$ due to weak localization effects. The typical scale $t_{\mathrm{E}}=$ $\lambda^{-1}|\log \hbar|$ is the Ehrenfest time with $\lambda$ the classical Lyapunov exponent. These corrections do not invalidate the BGS conjecture since $\delta E \sim \hbar / t_{\mathrm{E}}$ is much larger than the mean lever spacing $\Delta$, the natural scale in which WD statistics holds. 
Another potential source of deviations from WD statistics is related to energy scales larger than $\hbar / t_{\mathrm{fl}}$, where $t_{\mathrm{ff}}$ is the typical time for the classical dynamic to become fully chaotic. In billiards it is just the flight time of a particle across the system. These corrections can be expressed analytically [10, 11] in terms of the spectral determinant of the Perron-Frobenious operator, which controls the relaxation of the classical density to the ergodic-universal limit described by random matrix theory. As for weak localization corrections, the associated energy scale is much larger than the mean level spacing and consequently does not really affect the validity of the BGS conjecture.

From the previous discussion it is clear that the main deviation from the semiclassical picture contained in the BGS conjecture is due to strong quantum localization effects, or as it is usually referred in the literature, Anderson localization. After this introduction some questions arise: Why in some quantum chaotic systems localization is only weak and in others is strong enough to make the system and insulator invalidating thus the BGS conjecture? Are chaotic systems the only ones in which the BGS conjecture applies? If not so, is it possible to give a precise relation between classical motion and quantum features? And is it possible to define any other universality class in the context of non-random Hamiltonians?

In this paper we aim to address these questions by using ideas and techniques originally developed in the theory of disordered systems [12] such as the one-parameter scaling theory [13].

We start with a brief review that highlights some of the main ideas and results of this field. One of the main problems in the theory of disordered systems is the study of the role of quantum effects such as tunneling and interference on the transport properties of a particle in a random potential. In general, transport properties are strongly affected by both the dimensionality of the space, $d$, and the strength of disorder.

In two and lower dimensions destructive interference caused by backscattering produces exponential localization of the eigenstates in real space no matter the amount of disorder considered [12]. As a consequence, quantum transport is suppressed, the spectrum is uncorrelated (Poisson), and the system becomes an insulator. In three and higher dimensions there exists a metal-insulator transition for a critical amount of disorder. Thus for disorder below the critical one the wave functions are extended through the sample, namely, they are effectively represented by a superposition of plane waves with random phases. The Hamiltonian is accurately approximated by a random matrix with the appropriate symmetry, and the spectral correlations are given by WD statistics [14]. In the opposite limit, wave functions are exponentially localized as in $d \leq 2$, and Poisson statistics applies.

As in the quantum chaos case these universal features are restricted to long time scales (related to energy scales of the order of the mean level spacing) such that an initially localized wave-packet has already explored the whole phase space 
available. In other words, universality is related to a certain ergodic limit of the quantum dynamics. For shorter time scales the system has not yet relaxed to the ergodic limit and deviations from universality are expected.

These features can be understood within the framework of the one-parameter scaling theory [13]. A key concept in this theory is the dimensionless conductance $g$ introduced by Edwards and Thouless [15]. It is defined either as the sensitivity of a given quantum spectrum to a change of boundary conditions in units of the mean level spacing $\Delta \propto 1 / L^{d}$ or as $g=E_{\mathrm{c}} / \Delta$, where $E_{\mathrm{c}}$, the Thouless energy, is an energy scale related to the diffusion time to cross the sample. In the semiclassical limit $E_{\mathrm{c}}=\hbar D_{\text {cla }} / L^{2}$ ( $D_{\text {cla }}$ is the classical diffusion constant) and therefore, $g \propto L^{d-2}$. On the other hand, if the particle is exponentially localized, $g \propto \mathrm{e}^{-L / \xi}$, where $\xi$ is the localization length and $L$ is the system linear size.

The change of the dimensionless conductance with the system size is an indicator of localization. Qualitatively, in the $L \rightarrow \infty$ limit, $g \rightarrow \infty$ in a metal and $g \rightarrow 0$ in an insulator. In order to proceed it is useful to define

$$
\beta(g)=\frac{\partial \log g(L)}{\partial \log L},
$$

which describes the running of $g$ with the system size.

From the above definitions, $\beta(g)=d-2>0$ in a metal (without quantum corrections) and $\beta(g)=\log (g)<0$ in an insulator.

The one-parameter scaling is based on the following two simple assumptions:

- The $\beta(g)$ function is continuous and monotonous;

- The change in the conductance with the system size only depends on the conductance itself, which is a function of the system size.

We are now ready to analyze how localization corrections depend on the spatial dimensionality $d$ :

1. $d=1$. For $g \rightarrow \infty, \beta(g)=-1<0$. In the opposite limit $g \rightarrow 0, \beta(g)=$ $\log (g)<0$. Since $\beta(g)<0$ for any $g$, the dimensionless conductance always decreases with the system size and the system will be an insulator in the $L \rightarrow \infty$ no matter the amount of disorder.

2. $d=3$. For $g \rightarrow \infty, \beta(g)=1>0$. In the opposite limit however $g \rightarrow 0$, $\beta(g)=\log (g)<0$. Since $\beta(g)$ is continuous and monotonous there must be $g=g_{\mathrm{c}}$ such that $\beta\left(g_{\mathrm{c}}\right)=0$. At $g=g_{\mathrm{c}}$ the system undergoes a metalinsulator transition. The one-parameter scaling theory thus predicts a new window of universality characterized by a scale invariant dimensionless conductance $g=g_{\mathrm{c}}$. For a fixed disorder, if $g(L)>(<) g_{\mathrm{c}}$ then the system will flow to the metallic (insulator) limit as $L \rightarrow \infty$. A similar picture holds for $d>3$. The spectral correlations at the Anderson transition, usually referred to as critical statistics $[16,17]$, are scale invariant and intermediate between the prediction for a metal and for an insulator $[17,18]$. By scale invariant we mean that any spectral correlator utilized to describe the spectral properties of the disordered Hamiltonian does not depend on the system size. 
Eigenfunctions at the Anderson transition are multifractals [19, 22] (for a review see [21, 22]), namely, their moments present an anomalous scaling, $\mathcal{P}_{q}=\int \mathrm{d}^{d} r|\psi(\boldsymbol{r})|^{2 q} \propto L^{-D_{q}(q-1)}$ with respect to the sample size $L$, where $D_{q}$ is a set of exponents describing the Anderson transition.

3. $d=2$. For $g \rightarrow \infty, \beta(g)=0$ but for $g \rightarrow 0, \beta(g)=\log (g)<0$. In this case, since $\beta(g)=0$, the semiclassical analysis is not enough to determine the localization properties in the thermodynamic limit. We have to determine, whether $\beta(g)$ is positive or negative (or it remains zero) once quantum interference effects (weak localization corrections) are included. Technically one uses standard perturbation theory to compute one loop quantum corrections to the classical diffusion constant included in the definition of the dimensionless conductance. The result of this calculation is that $\beta(g) \sim-a / g$ $(a>0)$ for $g \gg 1$. Therefore, $\beta(g)<0$ for all $g$ and, as in the $1 d$ case, all eigenstates are localized in the $L \rightarrow \infty$ for any amount of disorder. We note that the negative sign of the weak localization correction is not universal, it depends on the details of the Hamiltonian. We shall see that if the classical diffusion is anomalous, weak localization corrections renormalize the value of the classical diffusion constant, but the semiclassical critical point $\beta(g)=0$ is not modified.

A few comments are in order:

1. The Anderson transition in a disordered conductor is a consequence of a highly nontrivial interplay between quantum destructive interference effects and quantum tunneling. In low dimensions, $d \approx 2$, weak quantum destructive interference effects induce the Anderson transition. Analytical results are available based on perturbation theory around the metallic state [23, 24]. In high dimensions, $d \gg 2$, quantum tunneling is dominant and the locator expansion [12] or the self-consistent formalism [25] can be utilized to describe the transition. We note that in these papers corrections due to interference of different paths are neglected.

2. The above analysis is strictly valid only for time reversal invariant systems with no interactions. A strong magnetic field or a spin-orbit interaction may induce a metal-insulator transition in two dimensions.

To summarize: According to the BGS conjecture, the level statistics of quantum systems whose classical counterpart is chaotic are described by WD statistics and eigenfunctions are delocalized in momentum space. According to the BTG conjecture, the level statistics of quantum systems, whose classical counterpart is integrable, are described by the Poisson statistics and the eigenstates are localized due to symmetry constraints. Strong localization effects typically invalidate the relation between classical chaos and WD statistics.

In the context of disordered systems the one-parameter scaling theory provides a valuable framework to understand localization effects. It predicts three universality classes: For $g \rightarrow \infty$ the system is a metal, eigenfunctions are delo- 
calized in real space and the spectrum is correlated according to WD statistics. For $g \rightarrow 0$ the system is an insulator, eigenfunctions are exponentially localized and the spectrum is correlated according to the Poisson statistics. For $g=g_{\mathrm{c}}$ a metal-insulator transition takes place. The eigenstates are multifractal and the spectral correlations are universal (critical statistics) but different from WD and Poisson statistics.

The organization of the paper is as follows: In Sect. 2 we discuss under which conditions the one-parameter scaling theory can be applied to quantum chaos. Then we identify a variety of problems in quantum chaos which the oneparameter scaling theory may help solve. In Sect. 3 we tackle one of them: the universality class related to the Anderson transition in quantum chaos. Section 4 is devoted to conclussions. We test numerically the prediction of the one-parameter scaling theory about the Anderson transition in quantum chaos in a $3 d$ kicked rotor with a smooth potentials and in a $1 d$ kicked rotor with singularities.

\section{One-parameter scaling theory in quantum chaos}

In this section we explain how to adapt the one-parameter scaling theory to the context of quantum chaos. The absence of a true ensemble average in quantum chaos is in principle a major obstacle for a straightforward application of techniques and ideas originally developed in the field of disordered systems. Fortunately, the one-parameter scaling theory is an exception. The basic assumptions (continuity and monotonicity of the $\beta(g)$ function and its sole dependence on $g$ ) do not lie on any ensemble average. Moreover, the mean level spacing in the definition of the dimensionless conductance have a well-defined meaning without having to perform an ensemble average. The Thouless energy, which is related to the classical diffusion time through the sample, is typically understood as an average over many realizations. However this is not necessary. We just need to find a way to determine a typical and meaningful travel time through the sample. For instance, in a kicked system it is the time it takes to explore a momentum basis of a given size. The main problem in quantum chaos is that in mixed systems it will depend strongly on the initial conditions. If the particle is initially located in an integrable region the typical time will be larger than in a chaotic region since Kolmogorov-ArnoldMoser (KAM) barriers slow down transport in momentum space. Thus in order to define properly the dimensional conductance in quantum chaos we have to impose the restriction that the classical phase space must be homogeneous. In this way a travel time can be defined unambiguously.

Another issue that requires clarification is the space in which the localization problem is defined. In disordered systems there is always the real space. Typically one fixes the strength of the disordered potential (for instance the density of scatterers) and then study how the dimensional conductance varies with the system size. By contrast, in quantum chaos the localization problem is typically defined in momentum space. Therefore, the Thouless time is defined as the time that a 
wave packet needs to explore a finite momentum basis of size $N$ (equivalently to the system size $L$ in disordered systems). As in disordered systems this can be estimated by computing the second moment $\left\langle p^{2}\right\rangle$ of the distribution.

The reason why localization is naturally defined in momentum space is related to the Noether theorem in classical mechanics, which states that for each classical symmetry there is a conserved quantity. If a system is classically integrable, the number of conserved quantities (canonical momenta) is equal to the dimensionality of the system. Semiclassically it is evident that each of these canonical momenta becomes a good quantum number, which labels the state. An integrable system is localized in momentum space since there exists a basis of good (momentum) quantum numbers, in which the Hamiltonian is diagonal and consequently the spectral correlations are Poisson-like as for an insulator. If the number of conserved momenta is smaller than the dimensionality of the space, the matrix representation of the Hamiltonian in any basis of momenta becomes non-diagonal and the spectrum is correlated to some degree. According to the BGS conjecture, in the case of no symmetry the spectral correlations are sufficiently strong that WD statistics applies. We note that this semiclassical picture does not take into account the strong localization effects that tend again to drive the system to the insulator limit.

To summarize: in quantum chaos the dimensionless conductance is a meaningful quantity provided that classical phase space is homogeneous. The localization problem in quantum chaos is naturally defined in momentum space.

The classical dynamics is quantum chaos and is by no means restricted to standard diffusion $\left\langle p^{2}\right\rangle=D t$. Different types of anomalous diffusion,

$$
\left\langle p^{2}\right\rangle \sim t^{\beta}
$$

with $\beta \neq 1$, may be relevant. The mean level spacing is in many cases given by $\Delta \sim 1 / N^{d}$, but there are important exceptions: (i) for periodic potentials, the Bloch theorem applies, the spectrum is continuous, and $\Delta=0$; (ii) for systems whose eigenstates are exponentially localized, $\Delta \neq 0$ even in the $N \rightarrow \infty$ limit; (iii) in systems with a singular continuous spectrum the scaling with the system size may be anomalous

$$
\Delta \propto N^{-d / d_{e}}
$$

$\left(d_{e} \neq 1\right)$. A precise definition of $d_{e}$ may depend on the system in question. In the Harper model $[6], d_{e} \approx 1 / 2$ stands for the Hausdorff dimension of the spectrum.

We are now ready to define the dimensionless conductance in quantum chaos. In the above-mentioned cases (i) and (ii), $g \rightarrow \infty$ (metal) and $g=0$ (insulator), respectively. In the case (iii) (including $d_{e}=1$ ), we define, with the help of Eqs. (3) and (4), the dimensionless conductance in quantum chaos,

$$
g(N)=\frac{E_{\mathrm{c}}}{\Delta}=N^{\gamma_{\text {clas }}}, \quad \gamma_{\text {clas }}=\frac{d}{d_{e}}-\frac{2}{\beta} .
$$


In disordered systems it is intuitively clear that if the strength of disorder is sufficiently strong, the insulator limit will be eventually reached. In quantum chaos it is not such evident as it may occur that the coupling constant of the model which plays the role of disorder never reaches this limit. This is a fact to take into account before making any statement about the existence of a metal-insulator transition in quantum chaos.

In order to study the change of the dimensionless conductance with the system size due to quantum effects, it is helpful to define the function $\beta(g)$ :

$$
\beta(g)=\frac{\partial \log g(L)}{\partial \log L} .
$$

If quantum effects are not important,

$$
\beta(g)=\gamma_{\text {clas }}=\frac{d}{d_{e}}-\frac{2}{\beta} .
$$

Based on the above expressions and under the assumptions of the one-parameter scaling theory we propose the following alternative definition of universality class in quantum chaos:

For systems such that $\gamma_{\text {clas }}>0$, eigenfunctions are delocalized as in a metal and the spectral correlations are universally described by WD statistics.

For systems such that $\gamma_{\text {clas }}<0$, eigenfunctions are localized as in an insulator and the spectral correlations are universally described by the Poisson statistics.

For systems such that $\gamma_{\text {clas }}=0$, eigenfunctions are multifractal as at the metal-insulator transition and the spectral correlations are universally described by critical statistics [16].

Assuming for the moment that the spectrum is not fractal, $d_{e}=1$, the one-parameter scaling theory predicts that if the classical motion is such that $\beta<2 / d$ the quantum properties are those of an insulator, no matter whether the classical dynamics is chaotic or not. For instance, for $d=3$, any classical motion leading to $\beta<2 / 3$, even if chaotic, will drive the quantum system to the insulator phase.

Another example is the $1 d$ kicked rotor with a smooth potential [7], classically $\left\langle p^{2}\right\rangle \propto t \rightarrow \gamma_{\text {clas }}=-1<0$. The one-parameter scaling predicts that the spectral correlations are described by the Poisson statistics as in an insulator even though the classical dynamics is chaotic. It is remarkable that by using scaling arguments dynamical localization can be predicted without having to map the problem onto a $1 d$ Anderson model.

Equation (6) also suggests that a metal-insulator transition $\gamma_{\text {clas }}=0$ is possible in less than two dimensions. In $1 d$, it is related to a superdiffusive classical motion such that $\beta=2 / d_{e} \geq 2$. For instance in non-conservative $1 d$ systems such as the kicked rotor $\left(d_{e}=1\right)$, the spectral correlations will be metal-like for $\beta>2$, similar to those of a metal-insulator transition for $\beta=2$ and, insulator-like for $\beta<2$. Therefore dynamical localization can be overcome, even in one dimension, if the classical diffusion is fast enough $(\beta \geq 2)$. 
We note that these conclusions are still restricted to the semiclassical approximation. Quantum effects such as destructive interference may slow down or even stop the quantum motion. However, the one-parameter scaling theory, as is the case of disordered systems, is capable to handle this situation as well. The way to proceed is to compute the Thouless energy not with the classical but with the quantum travel time to cross the sample. Assuming $\left\langle p^{2}\right\rangle_{\text {quan }} \propto t^{\beta_{q}}$, WD (Poisson) statistics applies if $\gamma_{q}=d / d_{e}-2 / \beta_{q}>(<) 0$. As an example we discuss the Harper model [6]. It is well known that this model undergoes a metal-insulator transition for a specific value of the coupling constant $(\lambda=2)$. We note that classically the system is integrable. The semiclassical dimensionless conductance cannot predict the transition. However, the quantum motion $\left\langle x^{2}\right\rangle \sim t^{2 d_{\mathrm{H}}}$ at the metal-insulator transition is diffusive where $d_{\mathrm{H}} \approx 0.5$ is the Hausdorff spectral dimension. According to our definition in Eq. (5), the "quantum" dimensionless conductance $g=g_{\mathrm{c}}$ is a constant independent of the system size. This is a signature of a metal-insulator transition. Therefore, our simple method predicts correctly the metal-insulator transition in this model as well.

\subsection{Stability of the semiclassical predictions}

From a more technical point of view the problem of the stability of the semiclassical predictions under quantum effects boils down to the way, in which the classical diffusion constant included in the definition of the dimensionless conductance gets renormalized by quantum corrections. For normal diffusion it is well known that quantum corrections (interference) may modify substantially the semiclassical results. For instance in a $2 d$ disordered system [22, 13], a simple calculation of the one-loop perturbative quantum correction shows that the diffusion constant is smaller than in the classical case, $\beta(g)$ becomes negative and the semiclassical metal-insulator transition is destroyed by quantum corrections.

However, in the case of classical anomalous diffusion we shall show that in certain circumstances semiclassical predictions are stable under quantum corrections. For the sake of simplicity we focus on the case $d=1$ and $d_{e}=1$. The extension of the results to other dimensions is straightforward. Our starting point is a classical diffusion process described by the following fractional Fokker-Planck equation:

$$
\left(\frac{\partial}{\partial t}-D_{\operatorname{clas}} \frac{\partial^{2 / \beta}}{\partial|p|^{2 / \beta}}\right) P(p, t)=\delta(p) \delta(t)
$$

(see [20] for the definition of fractional derivative), where $D_{\text {clas }}$ is the classical diffusion coefficient and $\beta$ is a real number. The moments of the distribution $P(p, t)$ (if well defined) are given by $\left\langle|p|^{k}\right\rangle \propto t^{\beta k / 2}$ with $k$ - real numbers. In analogy with the case of normal diffusion the propagator is given by $K_{0}(q, \omega=0)=\nu / D_{\text {clas }} q^{2 / \beta}$ with $\nu$ the spectral density.

The one-loop correction to the classical propagator due to interference effects takes the following form [20,22]: 


$$
K^{-1}(q)=K_{0}^{-1}(q)-\frac{(\pi \nu)^{2}}{2} \int \mathrm{d} k \frac{|q+k|^{2 / \beta}-|k|^{2 / \beta}}{|k|^{2 / \beta}} .
$$

The integral over $k$ is carried out, with the help of the following expansion $[23]$ :

$$
\frac{|q+k|^{2 / \beta}}{|k|^{2 / \beta}}-1 \simeq \begin{cases}\frac{2}{\beta} \frac{q k}{k^{2}}+\frac{1}{\beta} \frac{q^{2}}{k^{2}}+\frac{2}{\beta}\left(\frac{1}{\beta}-1\right)\left(\frac{q k}{k^{2}}\right)^{2}+\ldots, & q \ll k, \\ \frac{|q|^{2 / \beta}}{|k|^{2 / \beta}}, & q \gg k,\end{cases}
$$

where $L$, the system size, acts as an infrared cutoff.

The quantum diffusion constant $D_{\text {qua }}$ to one loop is easily obtained from Eqs. (9), (8),

$$
\begin{aligned}
& D_{\text {qua }}=D_{\text {clas }}-C L^{2 / \beta-1}, \quad 1<2 / \beta<2, \\
& D_{\text {qua }}=D_{\text {clas }}-C \log (q L), \quad 2 / \beta=1, \\
& D_{\text {qua }}=D_{\text {clas }}-C q^{1-2 / \beta}, \quad 0<2 / \beta<1,
\end{aligned}
$$

where $C$ is a different constant for each case.

The importance of the quantum effects depends strongly on the value of $2 / \beta$. In the region $1<2 / \beta<2$ quantum corrections diminish the value of the classical diffusion constant. These corrections, due to its dependence on the system size, grow as higher orders in perturbation theory are taken into account and eventually induce a transition to localization [22].

In the region $0<2 / \beta<1$ the correction is of higher order in $q$ than the classical term. This feature is not modified by higher orders in perturbation theory [22] and consequently the classical diffusion is not normalized.

The case $2 / \beta=1$, related to the metal-insulator transition, deserves a special consideration. The logarithmic behavior resembles superficially that of $2 d$ disordered system,

$$
D_{\text {qua }}=D_{\text {clas }}-C \ln (L / l) \text {. }
$$

However, there are essential differences. Comparing the two formulae, we see that in the case of anomalous diffusion the mean free path $l$ in the $2 d$ case is replaced by the inverse momentum $q^{-1}$. Therefore, the correction to the bare coupling constant, unlike the $2 D$ disordered conductor, is small for low momenta $q \sim 1 / L$. Qualitatively this implies the absence of eigenstate localization. For a rigorous proof based on the evaluation of $\beta(\mathrm{g})$ including higher order terms we refer to [22] and references therein.

Therefore, a Hamiltonian with classical dynamics, such as $2 / \beta=1$, should have quantum properties similar to that of a disordered conductor at the metalinsulator transition. If the classical diffusion is slower $(2 / \beta<1)$ quantum corrections will eventually induce a transition to localization. A final remark is in order. We recall that by dimension we do not mean the spatial dimension but the effective dimension in momentum space. A conservative one-dimensional (in space) system is always integrable, so no transition to delocalization can be observed. However, 
in momentum space the effective dimension is zero, just a point defined by energy conservation. But if energy is not conserved, as is the case in kicked rotors, the effective dimension in momentum space is the same as the spatial one, namely, the unity.

To summarize: We have identified three universality classes in quantum chaos: metal, insulator, and metal-insulator transitions. According to the one-parameter scaling theory these are the only windows of universality. If the classical diffusion is anomalous, we have shown in which circumstances semiclassical predictions are not substantially modified by quantum effects. Two ways to reach the metal-insulator transition in quantum chaos are identified: By classical anomalous diffusion such that $\gamma_{\text {clas }}=0$ or by quantum effects $\gamma_{q}=0$ in a classical Hamiltonian with normal diffusion and $\gamma_{\text {clas }}>0$.

\section{Scaling and the Anderson transition in quantum chaos}

We now test the predictions of the previous section in two specific models: A $3 d$ kicked rotor and a $1 d$ kicked rotor with a classical singularity. In the former the classical diffusion is normal. In the latter it is anomalous with an exponent which depends on the type of singularity. We shall show that by tuning the kicking strength (in $3 d$ ) or the type of singularity $(1 d)$ to a specific value we can induce a metal-insulator transition in quantum chaos. However the physical origin is slightly different in both situations. In $3 d$ it is caused by quantum destructive interference effects that slow down the classical chaotic motion, while in $1 d$ has its origin in the underlying classical anomalous diffusion. Finally, we mention other systems that, according to the classification scheme introduced in the previous section, also belong to the metal-insulator universality class.

\subsection{Anderson transition induced by classical anomalous diffusion}

Previously we determine by using the one-parameter scaling theory that classical superdiffusion may induce a metal-insulator transition even in $1 d$. In this section we provide numerical support to this theoretical prediction. In order to proceed we need to choose a system in which classical diffusion is anomalous. A suitable candidate is the kicked rotor with a non-analytical potential introduced in $[26]$,

$$
\mathcal{H}=\frac{p^{2}}{2}+V(q) \sum_{n} \delta(t-n T)
$$

with $V(q)=\epsilon|q|^{\alpha}$ and $V(q)=\epsilon \log (|q|) ; q \in[-\pi, \pi), \alpha \in[-1,1]$ and $\epsilon$ is a real number (for other type of singularities see $([27-29])$. The dynamical evolution over $T$ is dictated by the map: $p_{n+1}=p_{n}-\frac{\partial V\left(q_{n}\right)}{\partial q_{n}}, q_{n+1}=q_{n}+T p_{n+1}(\bmod 2 \pi)$.

We have evaluated the classical density of probability $P(p, t)$ and its moments $\left\langle p^{k}\right\rangle$ by evolving $10^{8}$ initial conditions uniformly distributed in $(q, p) \in$ $[-\pi, \pi) \times[-\pi, \pi)$. For $\alpha>0.5$ (see Fig. 1), the diffusion is normal and $P(p, t) \sim \mathrm{e}^{-c p^{2} / t} / \sqrt{t}(c$ a constant). However, for $-0.5<\alpha<0.5$ and $t>p$, 

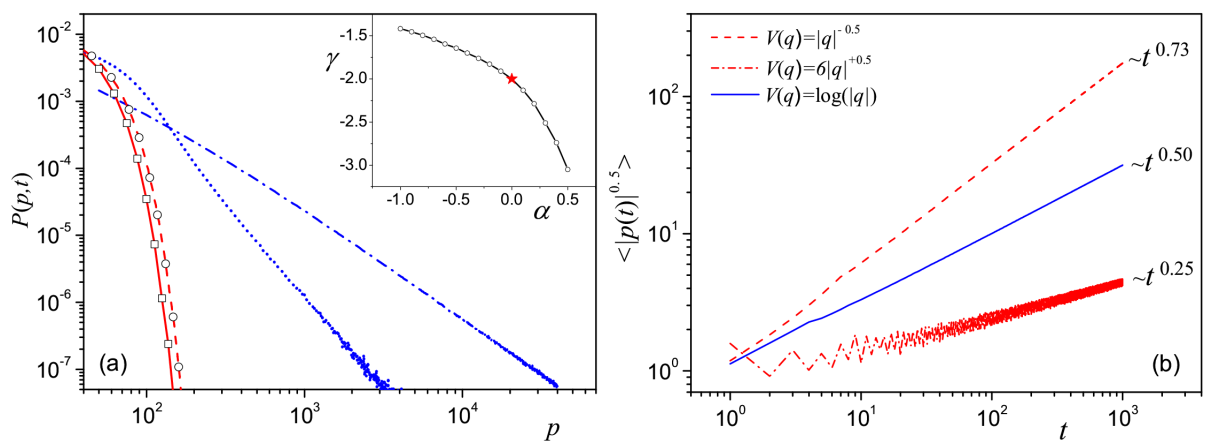

Fig. 1. (a) $P(p, t)$ versus $p$ (log scale). In all cases $t=2000$ except for $\alpha=-0.5$ where $t=40$. For $V(q)=|q|^{\alpha}$ with $\alpha=0.9$ (solid) and $V(q)=(|q|+0.005)^{0.4}$ (dash) the diffusion is normal (squares and circles are for best Gaussian fitting, respectively). However for $\alpha=0.4$ (dot) and $\alpha=-0.5$ (dash-dot), $P(p, t) \propto t p^{\gamma(\alpha)}$. Inset shows $\gamma$ as a function of $\alpha$. The star corresponds to $V(q)=\log (|q|)$. (b) Moments of the classical distribution for different $\alpha$ 's. As $\alpha$ increases classical diffusion is slower.

$P(p, t) \sim t p^{\gamma(\alpha)}$ has power-law tails (see inset in Fig. 1 for a relation between $\gamma$ and $\alpha$ ). Such tails are considered a signature of anomalous diffusion. As a consequence, the classical moments of the distribution scale as $\left\langle p^{k}\right\rangle \sim t^{(1-\alpha) k}$ (see Fig. 1) and the dimensionless conductance is given by

$$
\beta(g)=-\frac{\alpha}{1-\alpha} .
$$

According to the findings of the previous section, for $\alpha \leq 0$ quantum corrections do not modify qualitatively the semiclassical prediction for $\beta(g)$. Therefore, according to the one-parameter scaling theory we expect WD statistics for $\alpha<0$, the Poisson statistics for $\alpha>0$ (quantum corrections will only make the transition to localization faster) and an Anderson transition for $\alpha=0$ that corresponds naturally with the case of a log singularity.

We now compare these theoretical predictions with numerical results. In order to proceed we evaluate the quantum evolution operator $\mathcal{U}$ over a period $T$. After a period $T$, an initial state $\psi_{0}$ evolves to

$$
\psi(T)=\mathcal{U} \psi_{0}=\exp \left(-\mathrm{i} \hat{p}^{2} T / 4 \bar{h}\right) \exp (-\mathrm{i} V(\hat{q}) / \bar{h}) \exp \left(-\mathrm{i} \hat{p}^{2} T / 4 \bar{h}\right) \psi_{0},
$$

where $\hat{p}$ and $\hat{q}$ stand for the usual momentum and position operator. The spectrum was obtained by solving the eigenvalue problem $\mathcal{U} \Psi_{n}=\exp \left(-\mathrm{i} \kappa_{n} / \hbar\right) \Psi_{n}$, where $\Psi_{n}$ is an eigenstate of $\mathcal{U}$ with eigenvalue $\kappa_{n}$. We express the evolution operator in a matrix form $\langle m|\mathcal{U}| n\rangle$ in the basis of the momentum eigenstates $\left\{|n\rangle=\mathrm{e}^{\mathrm{in} \theta} / \sqrt{2 \pi}\right\}$. The resulting matrix (for $N$ odd) then reads

$$
\langle m|\mathcal{U}| n\rangle=\frac{1}{N} \mathrm{e}^{-\mathrm{i} 2 \pi M n^{2} / N} \sum_{l} \mathrm{e}^{\mathrm{i} \phi(l, m, n)},
$$


where $\phi(l, m, n)=2 \pi\left(l+\theta_{0}\right)(m-n) / N-\mathrm{i} V\left(2 \pi\left(l+\theta_{0}\right) / N\right), l=-(N-1) / 2, \ldots$ $(N-1) / 2$ and $0 \leq \theta_{0} \leq 1 ; \theta_{0}$ is a parameter depending on the boundary conditions $\left(\theta_{0}=0\right.$ for periodic boundary conditions). The eigenvalues and eigenvectors of $\mathcal{U}$ can now be computed by using standard diagonalization techniques.

We first analyze the moments of the quantum density of probability, $\left\langle p^{k}\right\rangle$. In Fig. $2 b$ it is observed that in agreement with the perturbative analysis above the classical time dependence is not modified by quantum corrections. However, the diffusion constant is smaller in the quantum case due to quantum de-
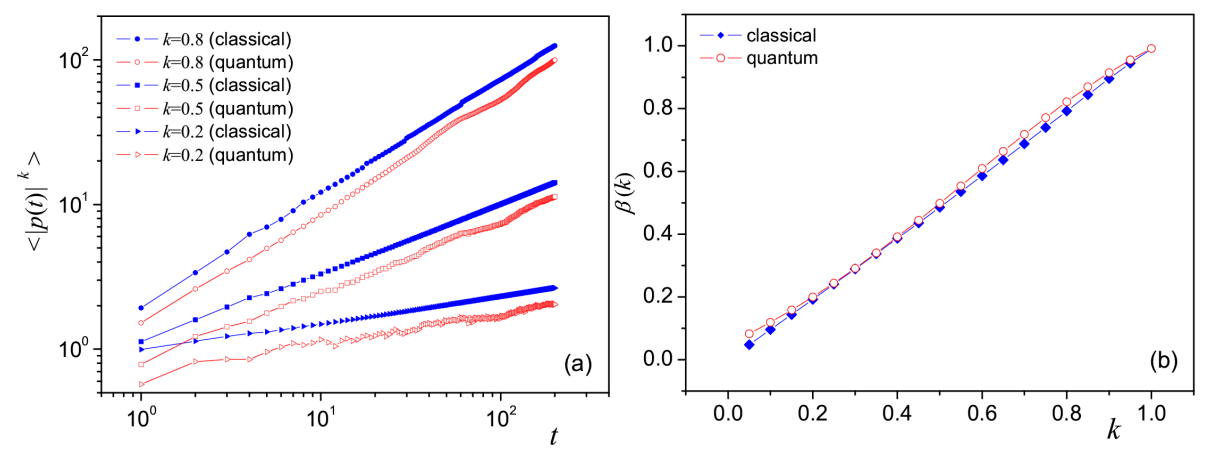

Fig. 2. Comparison of the classical and quantum time dependence of the moments $\left\langle|p|^{k}\right\rangle \propto t^{\beta(k)}$ for the case of a logarithmic singularity $(\alpha=0)$ related to the metalinsulator transition. The classical time dependence is not modified by quantum corrections (b). However, the value of the diffusion constant is smaller in the quantum case (a). This is an indication that quantum interference effects slow down the classical motion but cannot induce full dynamical localization. The same conclusions apply for $\alpha<0$.

structive interference effects (Fig. 2a). Similar results are obtained for $\alpha<0$. For $\alpha>0$ quantum corrections can only make the transition to localization faster. In conclusion, for $\alpha \leq 0$ the scaling of the dimensionless conductance does not change with respect to the semiclassical limit $g \propto L^{-\alpha}$, so a genuine metal-insulator transition is expected at $\alpha=0$.

The analysis of the level statistics confirms this theoretical prediction. The level spacing distribution $P(s)$ (see Fig. 3) tends to Poisson (WD) for $\alpha>0$ $(\alpha<0)$. As $\alpha$ approaches zero it is much more difficult to observe the transition to either WD or Poisson statistics. The reason for that is simple. The dimensionless conductance goes as $g(L) \propto L^{-\alpha}$ but the universal WD (Poisson) limit is only expected for $g \rightarrow \infty(g \rightarrow 0)$. Therefore, as we approach the transition $\alpha=0$, the universal limits are reached very slowly.

We now study the case of a potential with log singularity which according to our prediction is related to the Anderson transition. In order to proceed we also evaluate long range correlators as the spectral rigidity $\Delta_{3}(L)=\frac{2}{L^{4}} \int_{0}^{L}\left(L^{3}-\right.$ 


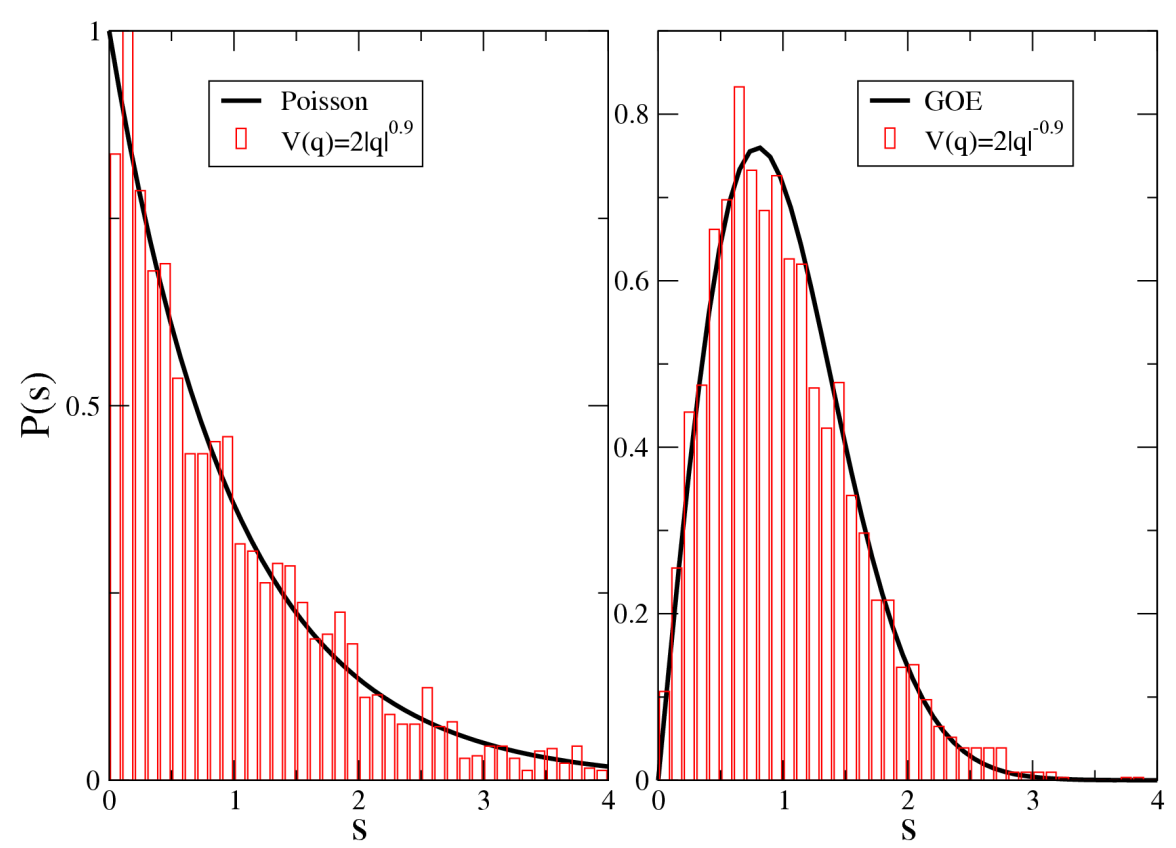

Fig. 3. The level spacing distribution $P(s)$ in units of the mean level spacing $s=$ $\left(E_{i}-E_{i-1}\right) / \Delta$. A transition is observed from the Poisson to WD statistics (keeping $\epsilon$ constant) as $\alpha$ goes from negative to positive. In both cases $N=3100$.

$\left.2 L^{2} x+x^{3}\right) \Sigma / 2^{2}(x) \mathrm{d} x$, where $\Sigma^{2}(L)=\left\langle L^{2}\right\rangle-\langle L\rangle^{2}=L+2 \int_{0}^{L}(L-s) R_{2}(s) \mathrm{d} s$ is the number variance and $R_{2}(s)$ is the two-level spectral correlations function. The $R_{2}(s)$ associated to this type of motion has been evaluated in Ref. [28] for systems with broken time reversal invariance,

$$
R_{2}(s)=-K^{2}(s)=-\frac{\pi^{2} h^{2}}{4} \frac{\sin ^{2}(\pi s)}{\sinh ^{2}\left(\pi^{2} h s / 2\right)},
$$

where $h \ll 1$ is related to $\epsilon$ by $h=1 / \epsilon \ll 1$. In Fig. 4 we show $\Delta_{3}(L)$ for different $\epsilon$ 's and $N=3099$. The time reversal invariance in Eq. (16) is broken by setting $T=2 \pi \beta$ with $\beta$ an irrational number. As observed in Fig. 4 (left), $\Delta_{3}(L)$ is asymptotically linear, this feature is typical of a disordered conductor at the metal-insulator transition. Moreover, the agreement with the analytical prediction based on Eq. (17) is excellent. We remark that the value of $h$ best fitting the numerical result is within $5 \%$ of the analytical estimate $h=1 / \epsilon$. We have repeated the calculation keeping the time reversal invariance $(T=2 \pi M / N)$ of Eq. (17) (Fig. 4, right). We do not have in this case an analytical result to compare with, but $\Delta_{3}(L)$ is also asymptotically linear as in the previous case.

These numerical results clearly support the analytical results of the previous section. Three and only three universality classes are observed: metal, insulator, and metal-insulator transition. Finally, we note that the novel semiclassical char- 


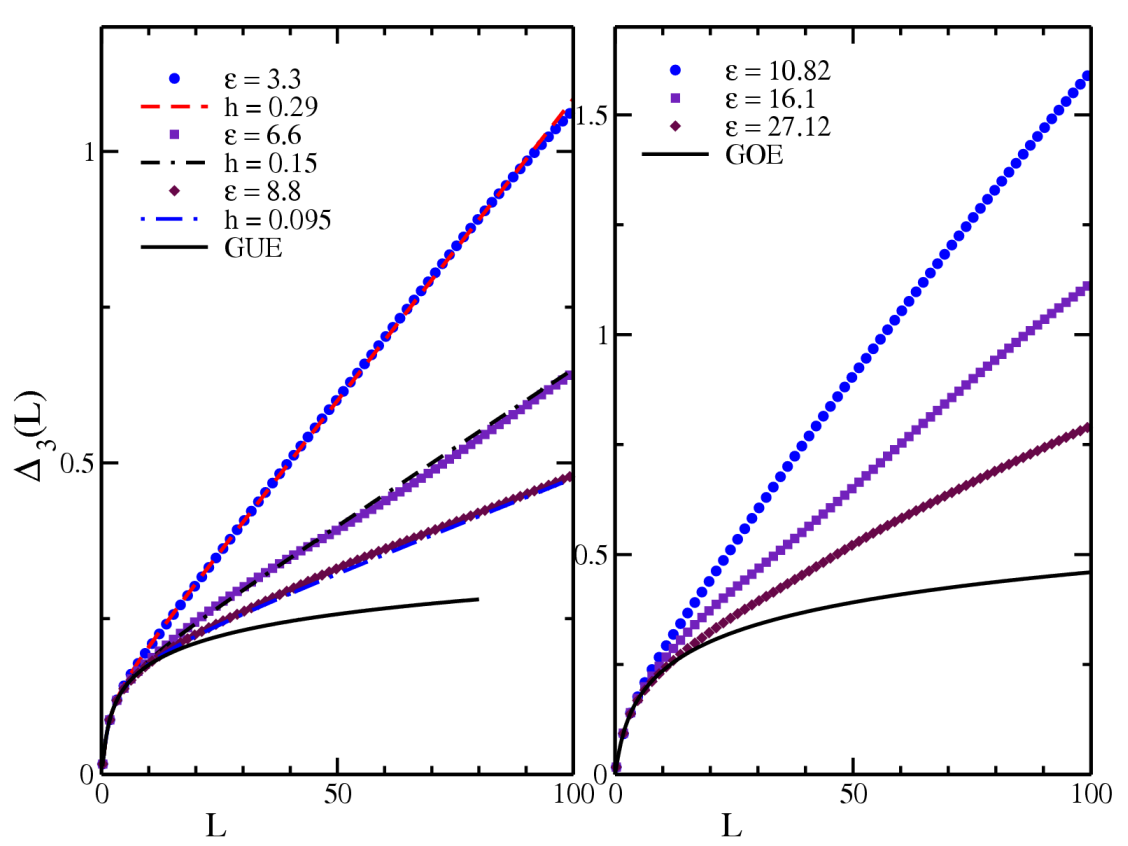

Fig. 4. The spectral rigidity $\Delta_{3}(L)$ versus $L$ for $V(q)=\epsilon \log (q)$. The right (left) part corresponds to the case of (broken) time reversal invariance. The linear behavior for $L \gg 1$ is a signature of the Anderson transition. The slashed lines in the case of broken time reversal invariance are the analytical prediction $\epsilon \approx 1 / h$ for this type of classical anomalous diffusion.

acterization of the metal-insulator transition proposed in this paper may be of interest for experimental studies about Anderson localization, as it enlarges the number of physical systems in which this phenomenon can be studied.

\subsection{Anderson transition induced by quantum fluctuations}

We now study the other route to the Anderson transition, namely, through quantum destructive interference effects that slow down an otherwise classically chaotic motion.

Our starting point is again the kicked rotor introduced in the previous section. However, this time it is defined in $3 d$ and with a smooth potential $V\left(\theta_{1}, \theta_{2}, \theta_{3}\right)=k \cos \left(\theta_{1}\right) \cos \left(\theta_{2}\right) \cos \left(\theta_{3}\right)$, the classical diffusion in momentum space is thus expected to be normal provided that the classical space is fully chaotic. We note if the spectrum is not fractal $\left(d_{e}=1\right)$ and the diffusion is normal, the dimensionality must be larger than two in order to observe a metal-insulator transition.

In Fig. $5 \mathrm{a}$ it is shown that as was expected classical motion is diffusive $\left\langle p^{2}\right\rangle \propto t$ in the range of $k$ of interest. Quantum dynamics depends strongly on $k$. In analogy with a $3 d$ disordered system we expect that strong Anderson localization effects stop the classical diffusion for sufficiently small $k$. In the opposite limit the 

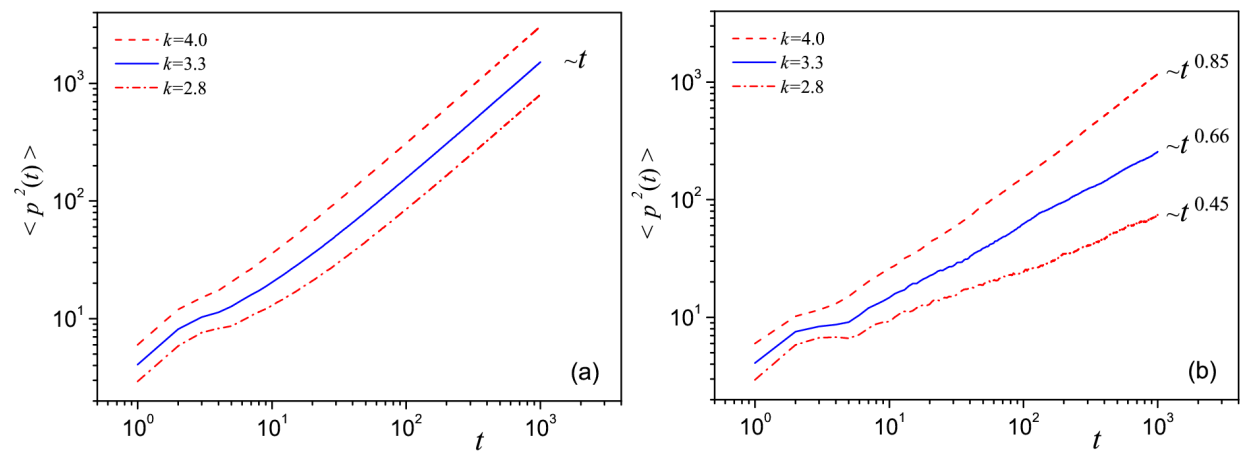

Fig. 5. Classical (a) and quantum (b) $\left\langle p^{2}\right\rangle$ for three different kicking values, above, below, and at the transition $k=k_{\mathrm{c}} \approx 3.3$ in the $3 d$ kicked rotor with a smooth potential. Classical diffusion is normal however quantum destructive interference slow down and eventually arrest the diffusion for $k<k_{\mathrm{c}}$. In agreement with the one-parameter scaling theory, at $k=k_{\mathrm{c}},\left\langle p^{2}\right\rangle \propto t^{2 / 3}$.
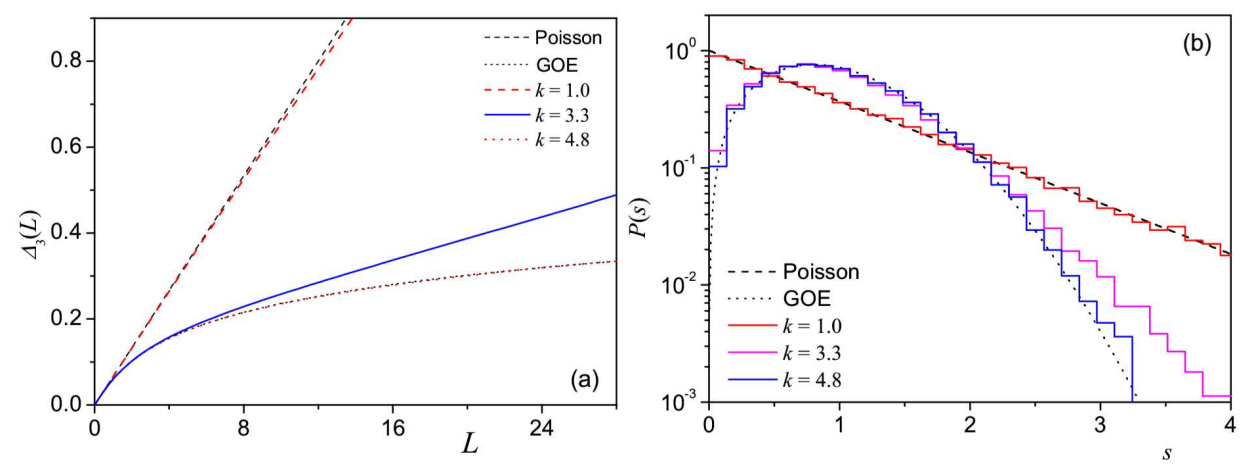

Fig. 6. The spectral rigidity $\Delta_{3}(L)$ versus $L$ (a) and level spacing $P(s)$ (b) for three different kicking values, above, below, and at the transition $k=k_{\mathrm{c}} \approx 3.3$ in the $3 d$ kicked rotor with a smooth potential. A transition from WD to Poisson statistics is clearly observed as the kicking strength crosses the critical value $k \approx k_{\mathrm{c}}$ from above. For $k \sim k_{\mathrm{c}}$ the level statistics have all the signatures of a metal-insulator transition such as linear $\Delta_{3}(L)$, level repulsion for $s \rightarrow 0$ as in a metal but exponential decay of $P(s)$ for $s>1$ as in an insulator. Although it is not shown, the spectrum is scale invariant at $k \approx k_{\mathrm{c}}$.

quantum effect should not modify substantially the classical motion. We carry out [30] a careful finite size scaling analysis in order to determine how localization corrections depend on $k$. A metal-insulator transition of purely quantum origin is located at $k=k_{\mathrm{c}} \sim 3.3$. Quantum diffusion becomes anomalous with $\beta=2 / 3$ (see Fig. 5b) in agreement with the prediction of the one-parameter scaling theory. In consequence the level statistics should be described by WD (Poisson) statistics in the limits $k \gg(\ll) k_{\mathrm{c}}$. 
As is shown in Fig. 6, the numerical results fully agree with these theoretical predictions. For $k=k_{\mathrm{c}}$ the spectral correlations have all the features of an $3 d$ Anderson transition including the numerical value of parameters such as the slope of the number variance $\approx 0.2$.

The kicked rotors studied in this paper are not the only ones that belong to the universality class of the metal-insulator transition. Other models that fall in it, at least for some value of the coupling constant, include Harper model [6], quantum exchange interval maps [31], Coulomb billiards [32], Kepler problem [33] and generalized kicked rotors [27, 28].

\section{Conclusions}

The main findings of this paper can be summarized as follows: For the first time we have adapted the one-parameter scaling theory to the context of quantum chaos. Then we have utilized it to determine the number of universality classes in quantum chaos and propose a more accurate definition of them. The one-parameter scaling theory has permitted us to give for the first time a precise definition of the universality class related to the metal-insulator transition. We have identified two routes (semiclassical and purely quantum) to reach a metalinsulator transition in quantum chaos. In order to support our theoretical conclusions we have investigated numerically a $1 d$ kicked rotor with singularities, where the Anderson transition has a semiclassical origin related to classical superdiffusion and the $3 d$ kicked rotor with a smooth potential, where the transition to localization is induced by destructive interference effects. Our findings open the possibility of studying the metal-insulator transition experimentally in a much broader type of systems.

\section{Acknowledgments}

A.M.G. acknowledges financial support from a Marie Curie Outgoing Action, contract MOIF-CT-2005-007300. J.W. is grateful to Professor C.-H. Lai for his encouragement and support, and acknowledges support from Defence Science and Technology Agency (DSTA) of Singapore under agreement of POD0613356.

\section{References}

[1] O. Bohigas, M.J. Gianonni, C. Schmit, Phys. Rev. Lett. 52, 1 (1984).

[2] S.W. McDonald, A.N. Kaufman, Phys. Rev. Lett. 42, 1189 (1979).

[3] G. Casati, F. Valz-Gris, I. Guarneri, Lett. Nuovo Cimento 28, 279 (1980).

[4] Chaos in Classical and Quantum Mechanics, Ed. M.C. Gutzwiller, Springer-Verlag, New York 1990; M.V. Berry, M. Tabor, Proc. R. Soc. Lond. A 356, 375 (1977).

[5] E.B. Bogomolny, B. Georgeot, M.-J. Giannoni, C. Schmit, Phys. Rev. Lett. 69, 1477 (1992). 
[6] S. Aubry, G. Andr, Ann. Israel Phys. Soc. 3, 133 (1980); M.Ya. Azbel, Zh. Eksp. Teor. Fiz. 46, 929 (1964); D.R. Hofstadter, Phys. Rev. B 14, 2239 (1976); D.J. Thouless, M. Kohmoto, M.P. Nightingale, M. den Nijs, Phys. Rev. Lett. 49, 405 (1982).

[7] G. Casati, B.V. Chirikov, F.M. Izrailev, J. Ford, in: Stochastic Behavior in Classical and Quantum Hamiltonian Systems, Eds. G. Casati, J. Ford, Lecture Notes in Physics, Vol. 93, Springer, Berlin 1979, p. 334; B.V. Chirikov, F.M. Izrailev, D.L. Shepelyansky, Sov. Sci. Rev. 2C, 209 (1981); B.V. Chirikov, Phys. Rep. 52, 265 (1979).

[8] S. Fishman, D.R. Grempel, R.E. Prange, Phys. Rev. Lett. 49, 509 (1982).

[9] I.L. Aleiner, A.I. Larkin, Phys. Rev. B 54, 14423 (1996); Phys. Rev. E 55, R1243 (1997).

[10] A.V. Andreev, B.L. Altshuler, Phys. Rev. Lett. 75, 902 (1995).

[11] A.V. Andreev, O. Agam, B.D. Simons, B.L. Altshuler, Phys. Rev. Lett. 76, 3947 (1996).

[12] P.W. Anderson, Phys. Rev. 109, 1492 (1958).

[13] E. Abrahams, P.W. Anderon, D.C. Licciardello, T.V. Ramakrishnan, Phys. Rev. Lett. 42, 673 (1979).

[14] M.L. Mehta, Random Matrices, 2nd ed., Academic Press, San Diego 1991.

[15] J.T. Edwards, D.J. Thouless, J. Phys. C 5, 807 (1972).

[16] V.E. Kravtsov, K.A. Muttalib, Phys. Rev. Lett. 79, 1913 (1997); S. Nishigaki, Phys. Rev. E 59, 2853 (1999).

[17] B.I. Shklovskii, B. Shapiro, B.R. Sears, P. Lambrianides, H.B. Shore, Phys. Rev. B 47, 11487 (1993)

[18] B.L. Altshuler, I.K. Zharekeshev, S.A. Kotochigova, B.I. Shklovskii, JETP 67, 62 (1988).

[19] M. Schreiber, H. Grussbach, Phys. Rev. Lett. 67, 607 (1991); H. Aoki, J. Phys. $C$ 16, L205 (1983).

[20] F. Evers, A.D. Mirlin, Phys. Rev. Lett. 84, 3690 (2000); E. Cuevas, M. Ortuño, V. Gasparian, A. Pérez-Garrido, Phys. Rev. Lett. 88, 016401 (2002); A.D. Mirlin, Y.V. Fyodorov, F.-M. Dittes, J. Quezada, T.H. Seligman, Phys. Rev. E 54, 3221 (1996); Y.V. Fyodorov, A.D. Mirlin, Phys. Rev. Lett. 67, 2405 (1991).

[21] M. Janssen, Int. J. Mod. Phys. B 8, 943 (1994); B. Huckestein, Rev. Mod. Phys. 67, 357 (1995); E. Cuevas, Phys. Rev. B 68, 184206 (2003).

[22] A.D. Mirlin, Phys. Rep. 326, 259 (2000).

[23] F. Wegner, Z. Phys. B 36, 209 (1980); F. Wegner, Z. Phys. B 25, 327 (1976).

[24] D. Vollhardt, P. Wolfle, Phys. Rev. Lett. 48, 699 (1982); ibid. 45, 842 (1980).

[25] R. Abou-Chacra, P. Anderson, D.J. Thouless, J. Phys. C, Solid State Phys. 6, 1734 (1973).

[26] A.M. Garcia-Garcia, J. Wang, Phys. Rev. Lett. 94, 244102 (2005).

[27] B. Hu, B. Li, J. Liu, Y. Gu, Phys. Rev. Lett. 82, 4224 (1999).

[28] J. Liu, W.T. Cheng, C.G. Cheng, Comm. Theor. Phys. 33, 15 (2000). 
[29] A.M. Garcia-Garcia, J. Wang, Phys. Rev. E 73, 036210 (2006).

[30] A.M. Garcia-Garcia, J. Wang, arXiv:0707.3964.

[31] E.B. Bogomolny, C. Schmit, Phys. Rev. Lett. 93, 254102 (2004); O. Giraud, J. Marklof, S. O'Keefe, J. Phys. A 37, L303 (2004).

[32] B.L. Altshuler, L.S. Levitov, Phys. Rep. 288, 487 (1997).

[33] D. Wintgen, H. Marxer, Phys. Rev. Lett. 60, 971 (1988). 